\title{
Supporting information: Droplet impact and spreading on inclined surfaces
}

Tushar Srivastava, Santosh Kumar Jena, and Sasidhar Kondaraju*

School of Mechanical Sciences, Indian Institute of Technology Bhubaneswar, Argul, Odisha 752050, India

Corresponding author* (email: sasidhar@iitbbs.ac.in)

\section{Contents}

Page

1 Front view images of droplet post-impact at different time intervals

2 Comparison of droplet spread values on different substrates

3 Side view snapshots of the droplet post-impact at different time intervals

4 Temporal evolution of droplet on different substrates for varying $\alpha$ and $v_{0}$ 


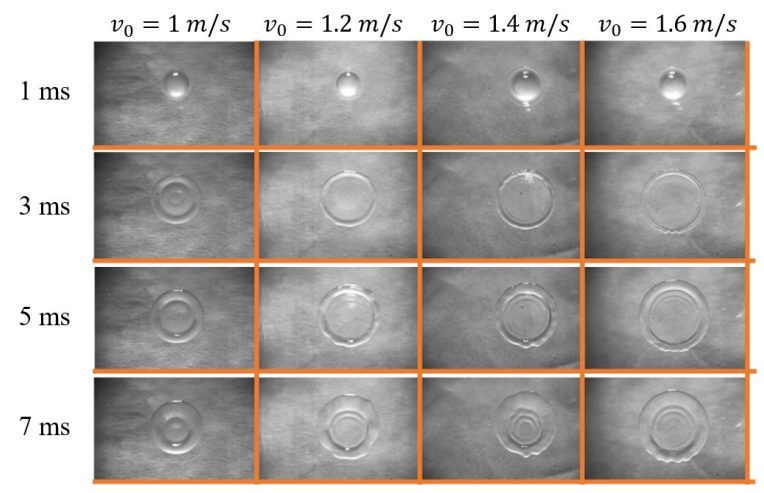

(a)

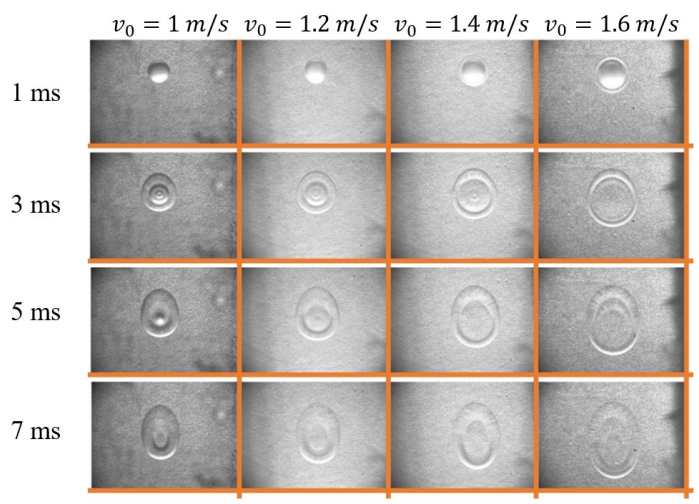

(c)

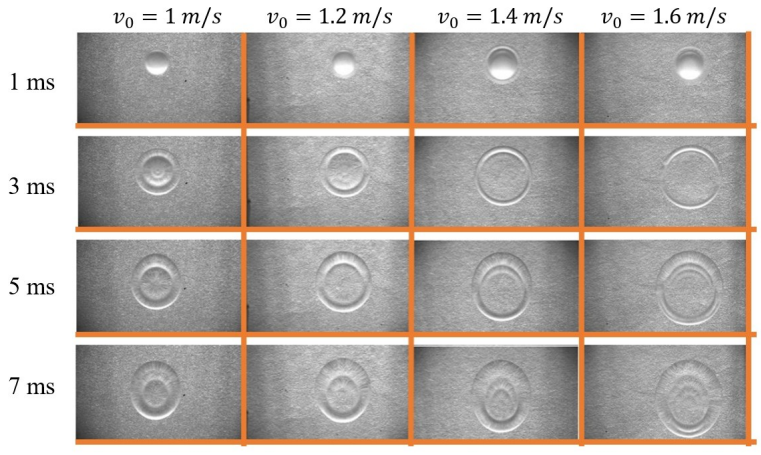

(b)

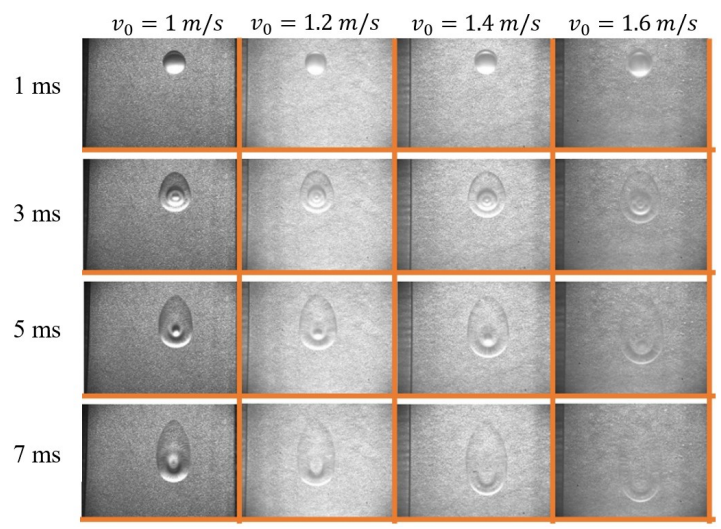

(d)

Figure s1: Front view images showing droplet shape post-impact on inclined PMMA at different time intervals and impact velocities when the substrate is inclined at: (a) $15^{\circ}$, (b) $30^{\circ}$, (c) $45^{\circ}$, and (d) $60^{\circ}$.

\section{Front view images of droplet post-impact at different time intervals}

Images in Figures s1 and s2 show the droplet shapes on PMMA and treated glass (TG) respectively as captured in a high-speed camera at $4000 \mathrm{fps}$ at separate time intervals: $1 \mathrm{~ms}, 3 \mathrm{~ms}, 5 \mathrm{~ms}$, and $7 \mathrm{~ms}$ after impact.

In the initial spreading phase, the droplet depicts close radial symmetry. As the surface inclination $\alpha$ increases, the asymmetries in the radial evolution of the droplet increase.

\section{Comparison of droplet spread values on different substrates}

Figure s3 plots spread values of water droplet post-impact on PMMA, TG, and Teflon when $\alpha$ is varied by keeping $v_{0}$ fixed. For low $\alpha$ droplet spread values on all substrates retract before reaching an equilibrium state. However, at high $\alpha$, the spread values for PMMA and TG continue to rise and the droplet is found to convert into a rivulet (Figure s4). The symbol $t$ represents time and $t=2.75 \mathrm{~ms}$ implies $2.75 \mathrm{~ms}$ after impact. 


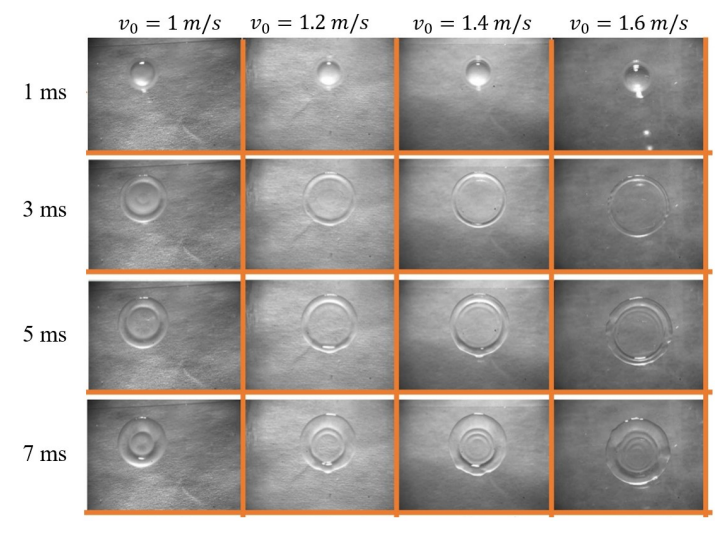

(a)

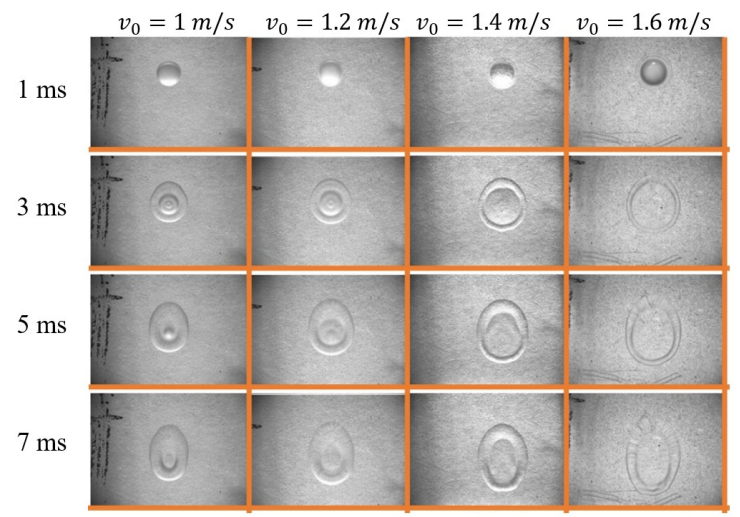

(c)

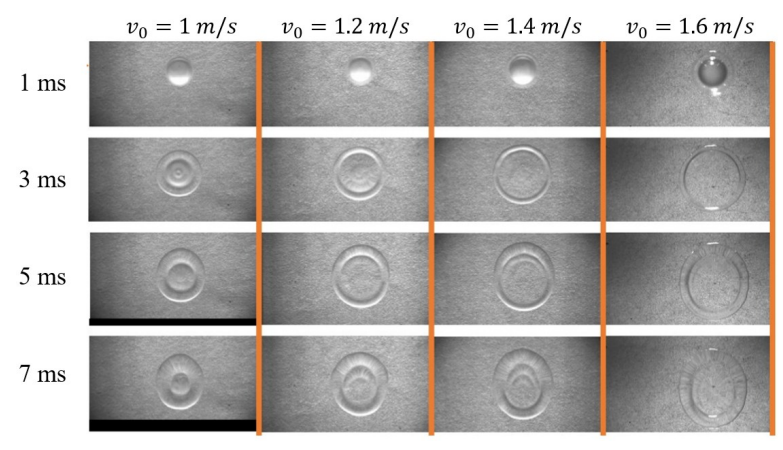

(b)

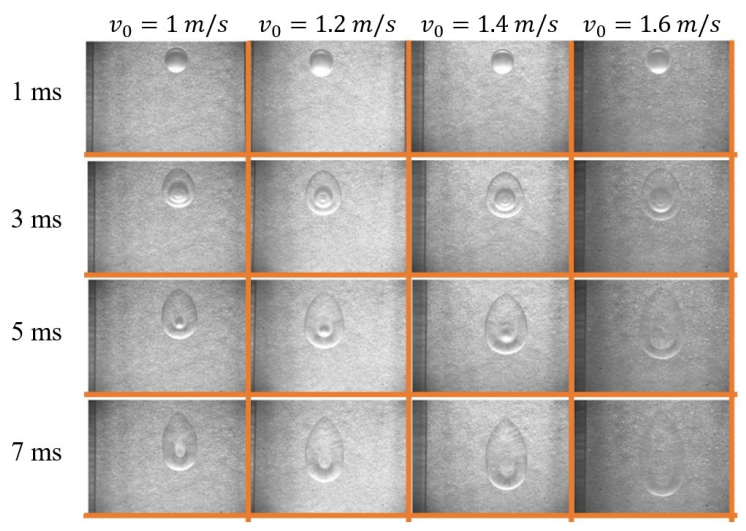

(d)

Figure s2: Images showing droplet shape post-impact on inclined treated glass (TG) at different time intervals and impact velocities when the substrate is inclined at: (a) $15^{\circ}$, (b) $30^{\circ}$, (c) $45^{\circ}$, and (d) $60^{\circ}$.

\section{Side view snapshots of the droplet post-impact at different time intervals}

Figure s5 depicts side-view snapshots (magnified) capturing the droplet spread post-impact on PMMA and Teflon (impact velocity $v_{0}=1 \mathrm{~m} / \mathrm{s}$ and surface inclination $\alpha=30^{\circ}$ ). The images were taken through a high-speed camera at 16000 fps to measure the dynamic contact angles during droplet spread. The time at which the droplet contacted the substrate is taken as 0 ms. Subsequent images were taken at $0.625 \mathrm{~ms}, 1.25 \mathrm{~ms}, 1.875 \mathrm{~ms}, 2.5 \mathrm{~ms}$, and $3.13 \mathrm{~ms}$ after impact.

\section{Temporal evolution of droplet on different substrates for varying $\alpha$ and $v_{0}$}

Figures s6 and s7 plot the temporal evolution of droplet spread behaviour obtained on different substrates when $\alpha$ and $v_{o}$ are varied. As observed, the spread behavior is similar for different impact velocities $v_{0}$ considered in the work. 


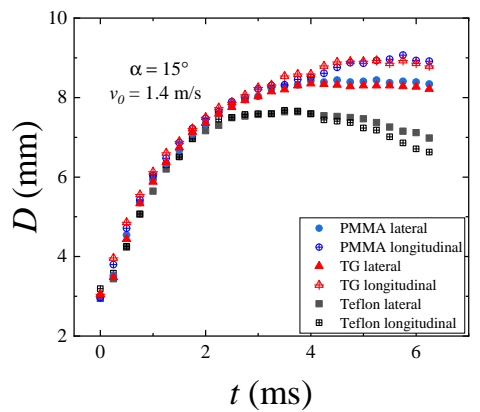

(a)

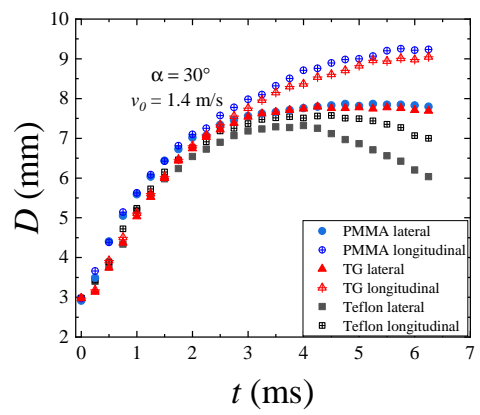

(b)

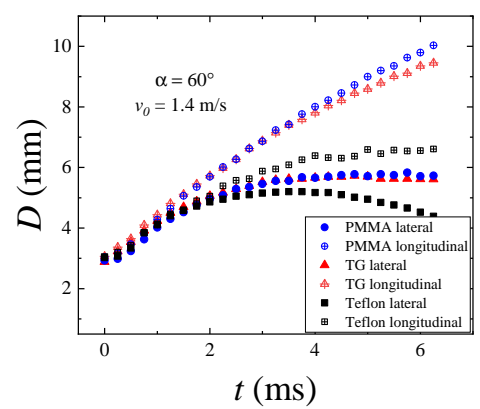

(c)

Figure s3: Plot comparing spread ' $D$ ' values of DIW droplet plotted against time ' $t$ ' post-impact on the three substrates PMMA, treated glass (TG), and Teflon in the longitudinal and lateral directions when the substrate inclination $\alpha$ is set to: (a) $15^{\circ}$, (b) $30^{\circ}$, and (c) $60^{\circ}$.

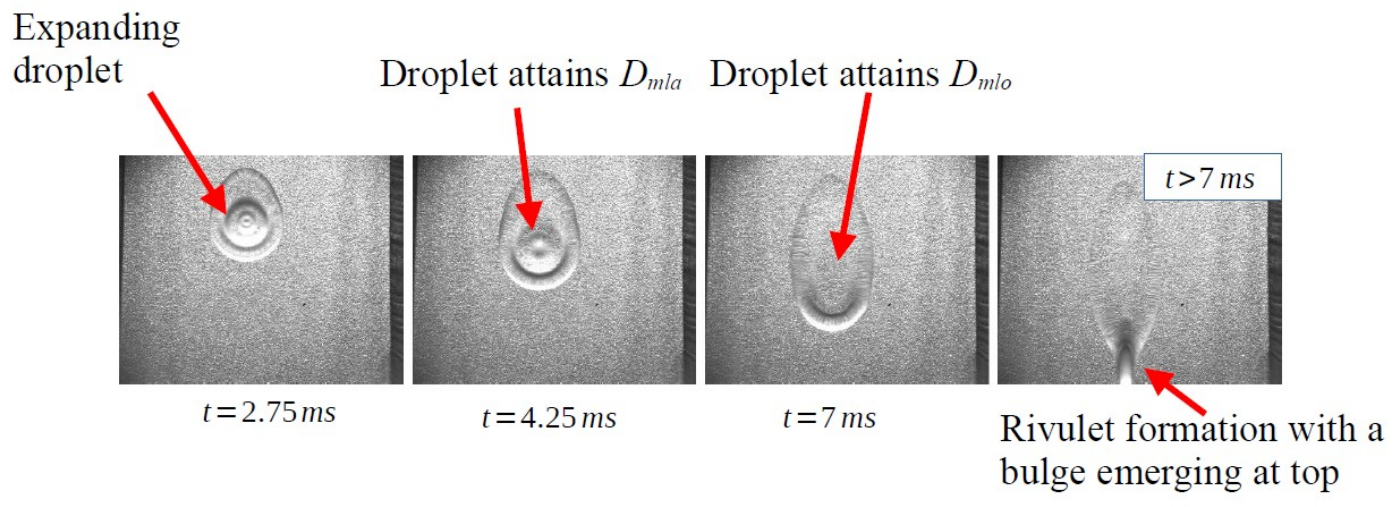

Figure s4: Droplet spread and features at different intervals of time $v_{0}=1.6 \mathrm{~m} / \mathrm{s}$ and $\alpha=60^{\circ}$. 


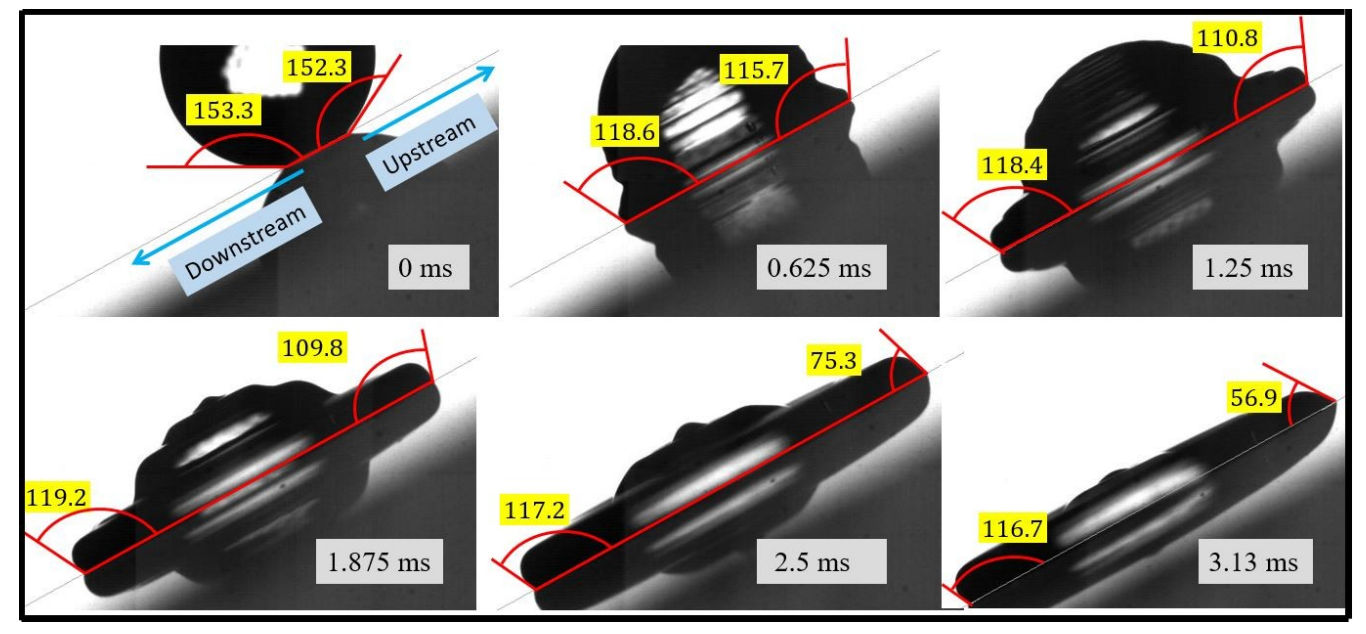

(a) PMMA

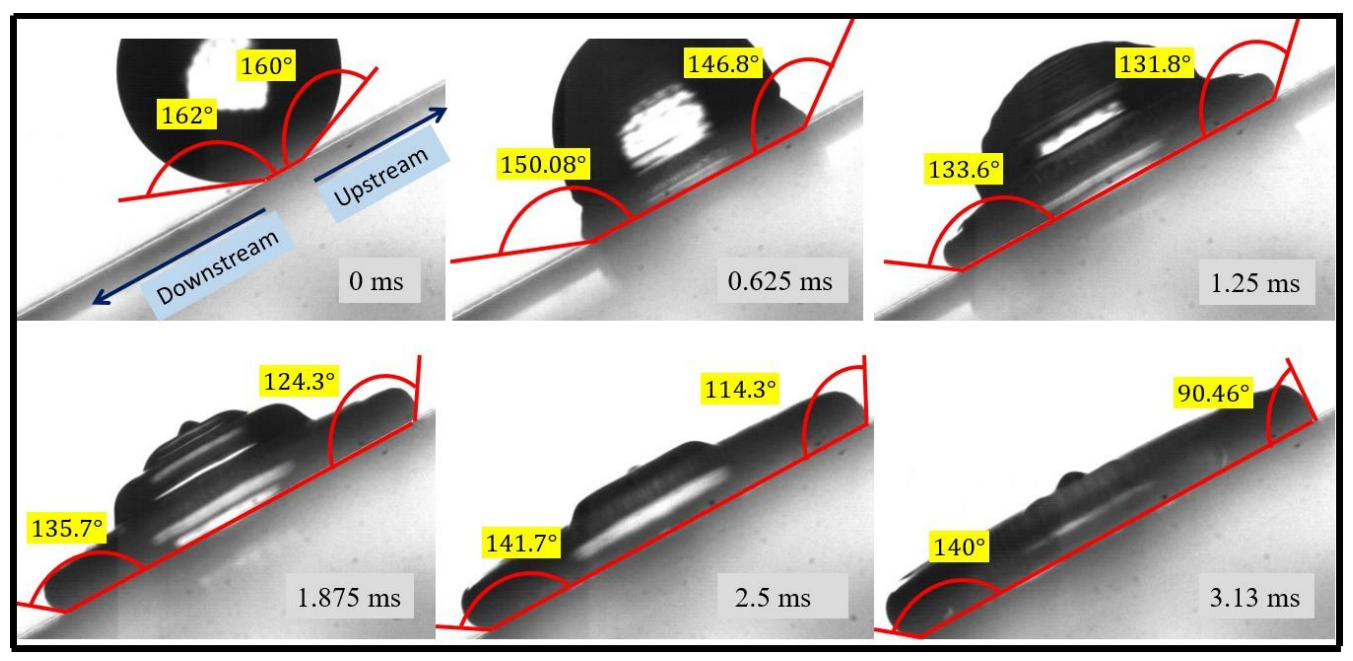

(b) Teflon

Figure s5: Plot depicting temporal side-view snapshots of water droplet spreading post-impact on the substrates: (a) PMMA, and (b) Teflon at impact velocity $v_{0}=1 \mathrm{~m} / \mathrm{s}$ and surface inclination $\alpha=30^{\circ}$. 


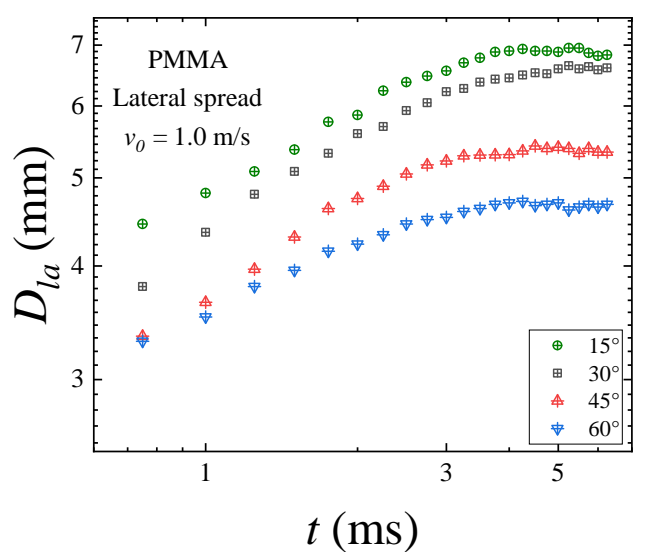

(a)

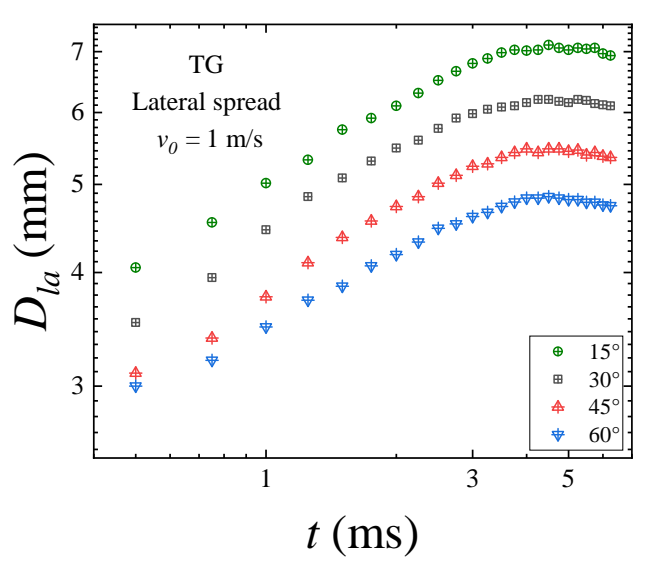

(c)

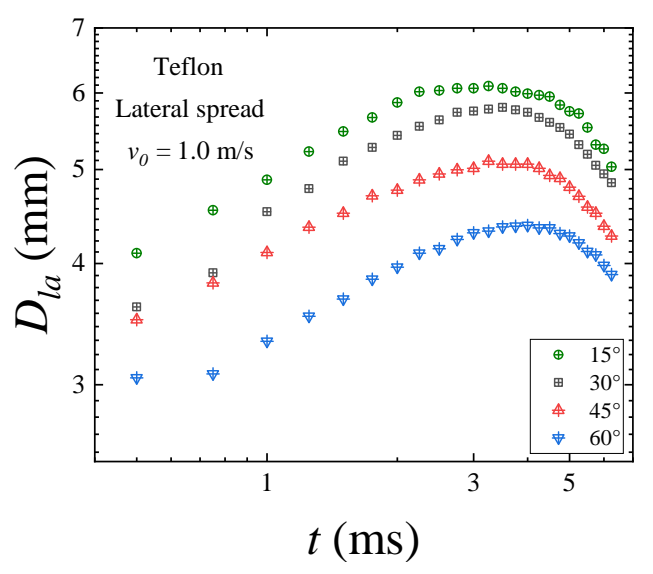

(e)

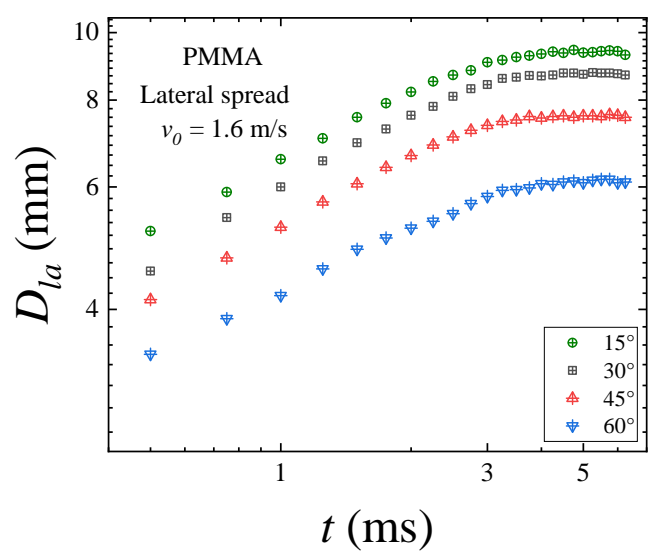

(b)

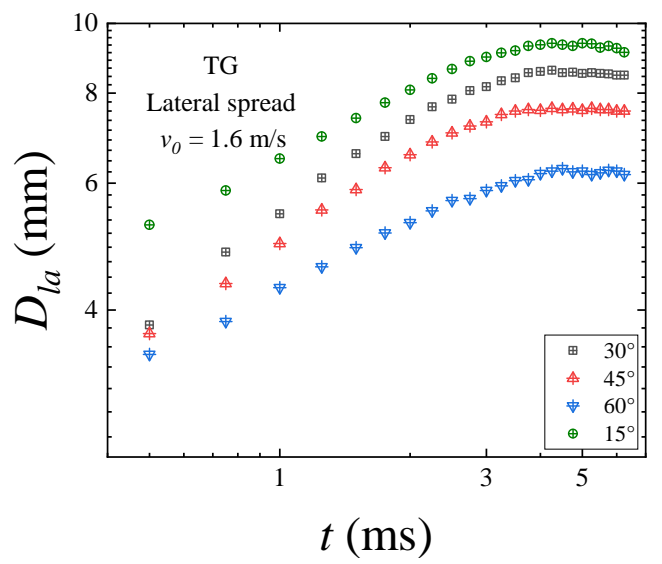

(d)

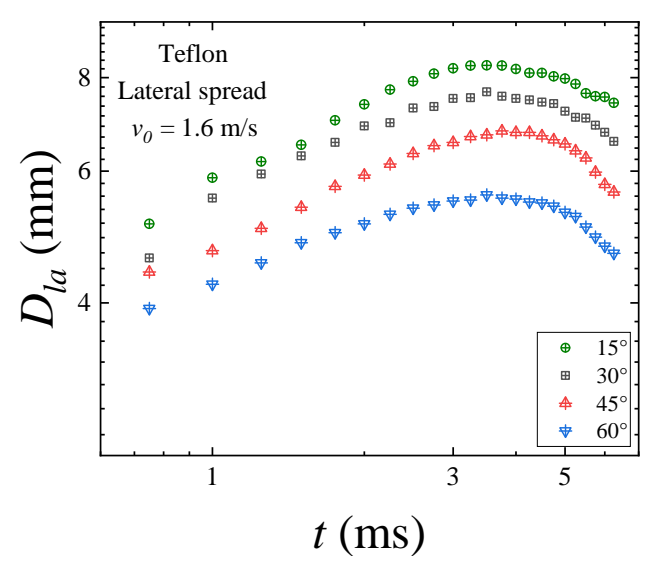

(f)

Figure s6: Temporal spread values in the lateral direction attained by DIW droplet post-impact on: (a) PMMA at impact velocity $v_{0}=1 \mathrm{~m} / \mathrm{s}$, (b) PMMA at impact velocity $v_{0}=1.6 \mathrm{~m} / \mathrm{s}$, (c) Treated glass (TG) at impact velocity $v_{0}=1 \mathrm{~m} / \mathrm{s}$, (d) Treated glass (TG) at impact velocity $v_{0}=1.6 \mathrm{~m} / \mathrm{s}$, (e) Teflon at impact velocity $v_{0}=1 \mathrm{~m} / \mathrm{s}$, and (f) Teflon at impact velocity $v_{0}=1.6 \mathrm{~m} / \mathrm{s}$ when substrate inclination $\alpha$ is varied. 


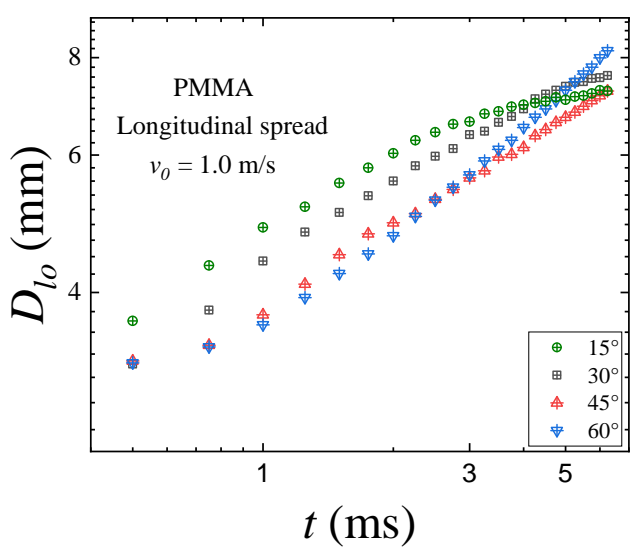

(a)

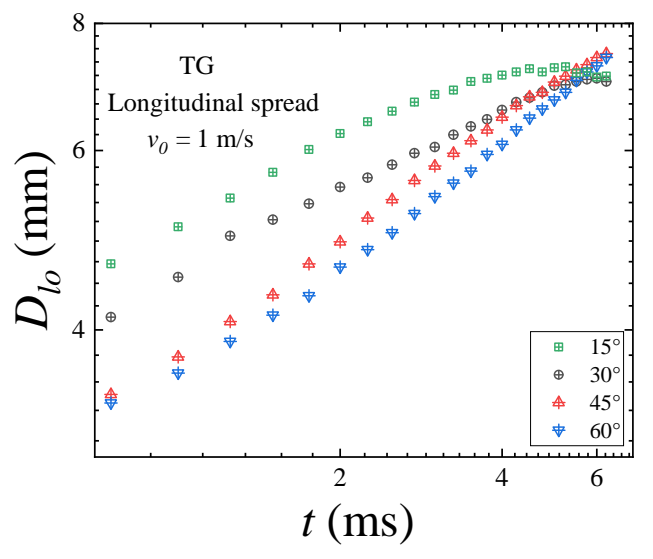

(c)

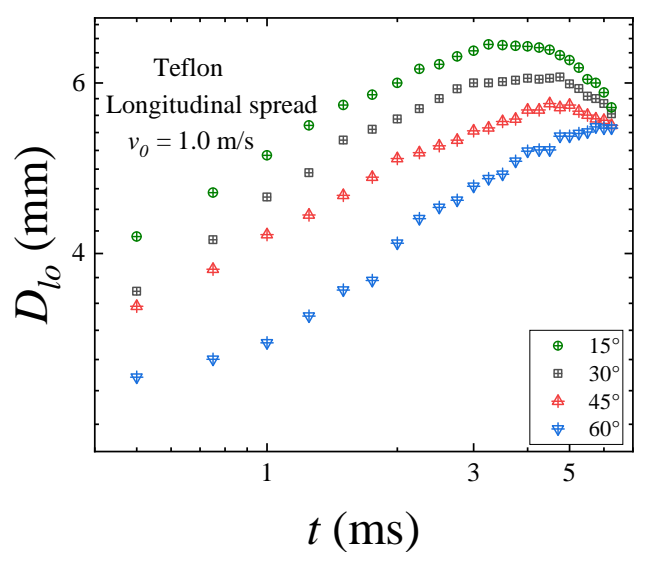

(e)

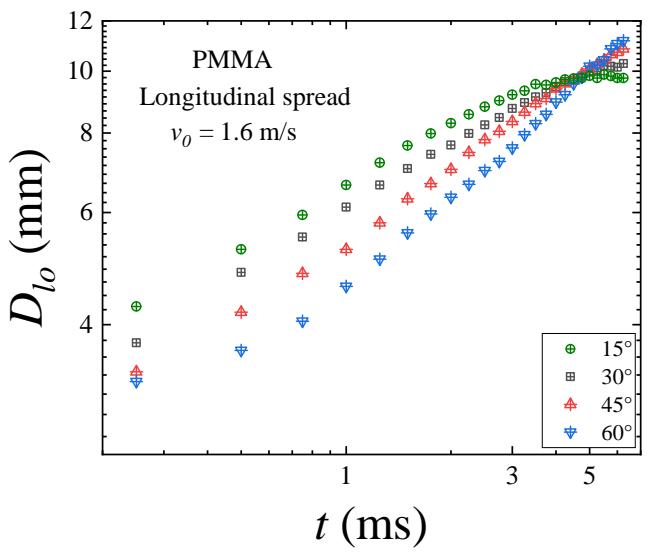

(b)

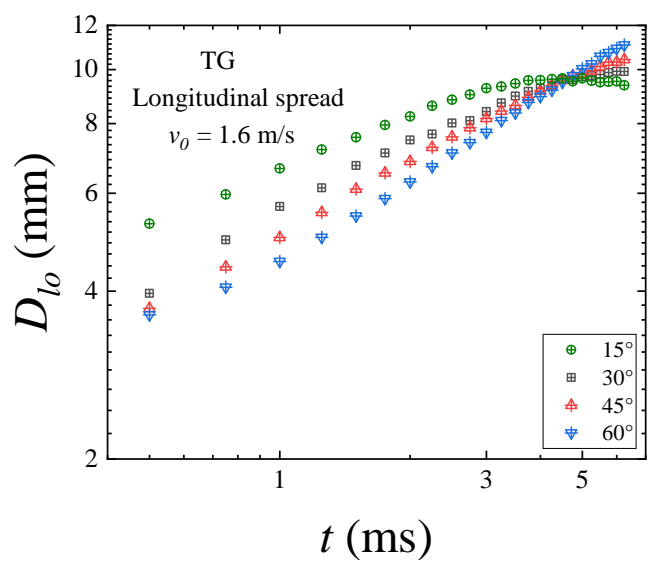

(d)

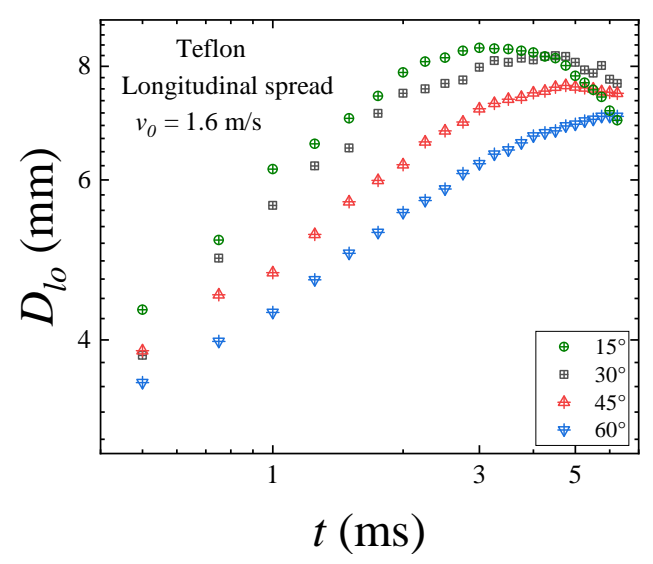

(f)

Figure s7: Temporal spread values in the longitudinal direction attained by DIW droplet post-impact on: (a) PMMA at impact velocity $v_{0}=1 \mathrm{~m} / \mathrm{s}$, (b) PMMA at impact velocity $v_{0}=1.6 \mathrm{~m} / \mathrm{s}$, (c) Treated glass (TG) at impact velocity $v_{0}=1 \mathrm{~m} / \mathrm{s}$, (d) Treated glass (TG) at impact velocity $v_{0}=1.6 \mathrm{~m} / \mathrm{s}$, (e) Teflon at impact velocity $v_{0}=1 \mathrm{~m} / \mathrm{s}$, and (f) Teflon at impact velocity $v_{0}=1.6$ $\mathrm{m} / \mathrm{s}$ when substrate inclination $\alpha$ is varied. 